\title{
AVALIAÇÃO DAS ÁREAS DE PRESERVAÇÃO PERMANENTE DO RIO MOGI GUAÇU, NO MUNICÍPIO DE PITANGUEIRAS - SP
}

LODO, Rafael Silveira ${ }^{1}$

ROSALEN, David Luciano ${ }^{2}$

RESUMO: A qualidade dos recursos hídricos de um manancial, além do seu uso, depende das atividades que se desenvolvem em suas margens. Dessa forma, a manutenção das Áreas de Preservação Permanente (APPs), conforme legislação vigente, é fundamental para contribuir com essa qualidade. O presente trabalho teve como objetivo verificar a invasão da agricultura em APPs localizadas ao longo do Rio Mogi Guaçu, município de Pitangueiras-SP. Através de imagens de satélites obtidas no sistema Google Earth, foi elaborado um mosaico não controlado e por meio de ferramentas de geoprocessamento, via ArcGIS, foi realizada a demarcação da largura do curso d'água e das APPs, assim como a identificação da invasão de APPs pela atividade agrícola. Os resultados obtidos indicaram que as APPs invadidas pela agricultura foram de aproximadamente 3,4\% em relação aos 429,3 ha de APPs ao longo do Rio Mogi Guaçu, presentes na área de estudo.

Palavras-chave: Preservação ambiental. Legislação Ambiental. SIG.

\section{DIAGNOSIS OF PERMANENT PRESERVATION AREAS MOGI GUAÇU RIVER, IN THE CITY OF PITANGUEIRAS-SP}

SUMMARY: The quality of water from a spring, in addition to their usedepends on the activities developed on its shores. Thus, the maintenance of the Areas of Permanent Preservation (APPs), according to current law, it is essential to contribute to this quality. This study aimed to verify the invasion of agriculture in APPslocated along the Rio Mogi Guaçu municipality Pitangueiras-SP. Through satellite images obtained from Google Earth system, we constructed a mosaic is not controlled by geoprocessing tools viaArcGIS Geographic Information System, was conducted to demarcate the width of the stream and of APPs, as well asidentification of the invasion of APPs by farming. The results indicated that the APP invaded by agriculture were approximately3.4\% compared to 429.3 ha of APPs along the Rio Mogi Guaçu,present in the study area.

Keywords: Environmental Conservation. Environmental Legislation. GIS.

\section{INTRODUÇÃO}

A qualidade dos recursos hídricos de um manancial, além de seu uso, depende das atividades que se desenvolvem em suas margens. Pode-se dizer que a qualidade está intimamente relacionada com o uso que se faz do solo em seu redor. Assim, em programas de preservação de recursos hídricos, deve-se considerar o todo (água e solo), de modo que o uso destes resultem no menor impacto possível sobre a qualidade da água. Desta forma, o disciplinamento do uso e da ocupação do solo numa bacia hidrográfica é altamente recomendado para a manutenção da qualidade dos recursos hídricos.

Objetivando disciplinar e limitar as interferências antrópicas sobre o meio ambiente, o artigo $2^{\circ}$ do Código Florestal Brasileiro - a Lei n. ${ }^{\circ}$ 4.771, de 15 de setembro de 1965 - contempla a criação das Áreas de Preservação Permanente (APP). Nessas áreas, é proibitivo a remoção da cobertura vegetal original, permitindo, assim, que ela possa exercer, em plenitude, suas funções ambientais (SOARES et al., 2002).

No início dos anos 90, na cidade do Rio de Janeiro, foi realizada a Conferência das Nações Unidas sobre o Meio Ambiente e o Desenvolvimento (ECO 92), na qual, os países com as maiores economias do

\footnotetext{
${ }^{1}$ Eng $^{\mathrm{o}}$ Agrônomo.

${ }^{2}$ Eng $^{\circ}$ Agrônomo, Professor Assistente Doutor, Universidade Estadual Paulista - UNESP/FCAV- Jaboticabal - Departamento de Engenharia Rural.
} 
planeta assumiram compromissos para melhorias do meio ambiente.

Acompanhando essa tendência, no Brasil, objetivando melhorias na preservação de seus recursos naturais, é estabelecida em dia 20 de março de 2002, a Resolução n 303 do Conselho Nacional do Meio Ambiente (CONAMA). Essa resolução fixou parâmetros, definições e limites referentes às Áreas de Preservação Permanente e adota, ainda que implicitamente, a bacia hidrográfica como unidade de sua aplicação, como cita o artigo $3^{\circ}$ dessa resolução (SOARES et al., 2002).

Dessa forma, o monitoramento das áreas de preservação permanente tem sido um grande desafio sob o aspecto técnico e econômico, pois os critérios de delimitação com base em levantamentos cadastrais exigem o envolvimento de pessoal especializado e de informações detalhadas da unidade espacial em análise.

Entretanto, o desenvolvimento de algoritmos específicos e sua respectiva incorporação ao conjunto de funções de Sistemas de Informação Geográfica (SIG) e a grande disponibilidade, hoje, de produtos de Sensoriamento Remoto, tornam possível o processamento rápido e eficiente dos dados necessários para a caracterização das variáveis morfométricas do terreno (OLIVEIRA, 2002). A utilização de SIGs possibilita a geração de bancos de dados codificados espacialmente, promovendo ajustes e cruzamentos simultâneos de um grande número de informações, possibilitando, ainda, a atualização dos bancos de dados para obtenção de novos mapas com rapidez e precisão (CAMPOS, 2001).

Dentro deste contexto, o presente trabalho teve como objetivo, avaliar a invasão de áreas de preservação permanente pela agricultura, ao longo do Rio Mogi Guaçu, no município de Pitangueiras - SP.

\section{MATERIAL E MÉTODOS ${ }^{3}$}

A área de estudo compreendeu o trecho do Rio Mogi Guaçu, no município de Pitangueiras - SP, cuja sede se situa nas proximidades da latitude $21^{\circ} 00^{\prime} 00^{\prime}$ ' $\mathrm{S}$, longitude $48^{\circ} 22^{\prime} 00^{\prime}$ ' $\mathrm{W}$ e altitude aproximada de $513 \mathrm{~m}$.

A Bacia Hidrográfica do Rio Mogi Guaçu pertence à Unidade de Gerenciamento de Recursos Hídricos do Mogi Guaçu, UGRHI-9 (Figura 1). Compreende uma área total de $17.460 \mathrm{~km}^{2}$, dos quais $14.653 \mathrm{~km}^{2}$ estão localizados na porção nordeste do Estado de São Paulo. Tem formato aproximadamente retangular e estende-se no sentido sudoeste - noroeste. Zancopé e Perez Filho (2006) destacam que o Rio Mogi Guaçu nasce no Morro do Curvado, no município sul - mineiro de Bom Repouso, localizado entre coordenadas as $22^{\circ} 30^{\prime} 00^{\prime}$ 'S e $46^{\circ} 08^{\prime} 00^{\prime}$ 'W, numa altitude aproximada de $1.510 \mathrm{~m}$, na região da Serra da Mantiqueira, próximo ao limite interestadual entre São Paulo e Minas Gerais, Sudeste Brasileiro.

Após escoar longitudinalmente por aproximados $530 \mathrm{~km}$, deságua no Rio Pardo a $483 \mathrm{~m}$ acima do

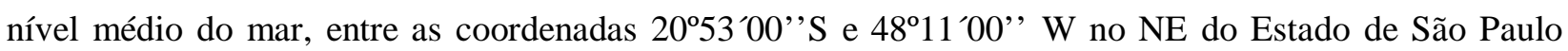
(CBH-MOGI).

${ }^{3}$ A citação de marcas não implica a recomendação destas pelos autores. 
Figura 1 - Localização da Unidade de Gerenciamento de Recursos Hídricos do Mogi Guaçu (UGRHI-9).

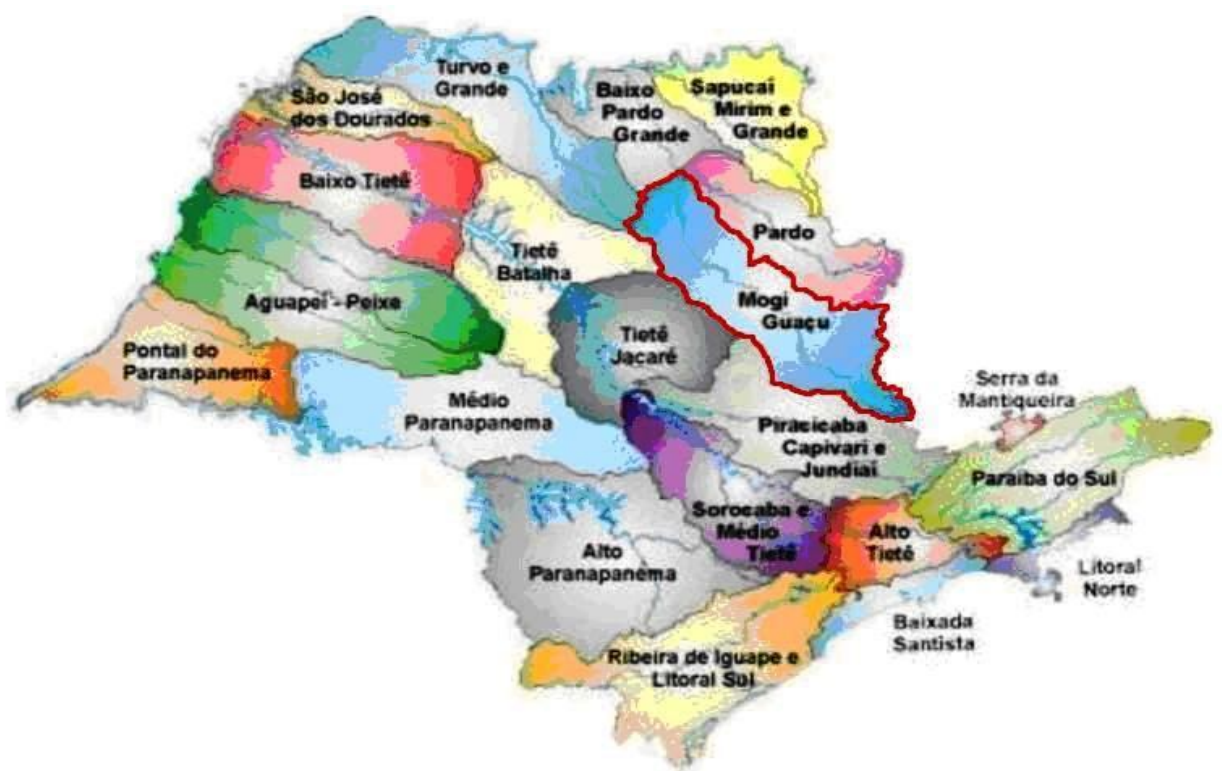

Para o desenvolvimento dos trabalhos, foram utilizadas três imagens de satélites distintas, sendo duas Digital Globe (DIGITAL GLOBE, 2011a e 2011b) e uma Spot (SPOT, 2011), obtidas através do sistema Google Earth v.6 (Tabela 01). Através do software ArcGIS v.9.3, foi montado um mosaico de imagens não controlado para a demarcação das APPs.

Tabela 1: Informações das imagens de satélite utilizadas para o estudo.

\begin{tabular}{lccc}
\hline \multicolumn{1}{c}{ Informações } & $\begin{array}{c}\text { Digital Globe } \\
\text { (Figura 3) }\end{array}$ & $\begin{array}{c}\text { Spot } \\
\text { (Figura 4) }\end{array}$ & $\begin{array}{c}\text { Digital Globe } \\
\text { (Figura 5) }\end{array}$ \\
\hline ID do catálogo: & 1010010005 C2B501 & $*$ & 1010010001 EC904 \\
Acq Data: & 20 de julho de 2007 & 22 de junho de 2009 & 19 de maio de 2003 \\
Centro de Lat / Long: & $-21.043^{\circ} /-48.273^{\circ}$ & $-21.0131^{\circ} /-47.9364^{\circ}$ & $-20,863^{\circ} /-48,153^{\circ}$ \\
Média Off Nadir & $1{ }^{\circ}$ & $*$ & $14^{\circ}$ \\
ângulo: & $82^{\circ}$ & $*$ & $295^{\circ}$ \\
Média Azimute Alvo: & QB02 & $*$ & QB02 \\
Sensor: & Pan_MS1 & $*$ & Pan_MS1 \\
Band Info: & & & \\
\hline
\end{tabular}

*Informações não fornecidas pela fonte.

Fonte: Google Earth (2011).

Foram amostradas 55 seções transversais a cada 500 metros, para a avaliação da largura do curso d'água ao longo do trecho citado para, dessa forma, possibilitar a determinação da faixa de APPs, através da ferramenta "régua" do Google Earth 6, conforme rege a legislação ambiental vigente no País no, artigo $2^{\circ}$ da Lei 4.771/65 (Tabela 2). 
Tabela 2: Largura de vegetação ciliar a ser mantida ou revegetada de acordo com a largura do curso de água, conforme artigo $2^{\circ}$ da Lei $4.771 / 65$.

\begin{tabular}{lc}
\hline \multicolumn{1}{c}{ Largura do curso d'água (m) } & Largura da APP $(\mathbf{m})$ \\
\hline Menor que 10 & 30 \\
De 10 a 50 & 50 \\
De 50 a 200 & 100 \\
De 200 a 600 & 200 \\
Maior que 600 & 500 \\
Nascentes & 50 \\
\hline
\end{tabular}

\section{RESULTADOS E DISCUSSÃO}

O Rio Mogi Guaçu entra na área de estudo nas proximidades das coordenadas $21^{\circ} 05^{\prime} 21^{\prime \prime} \mathrm{S}$ e $48^{\circ} 11^{\prime} 01^{\prime}$ 'W, numa altitude aproximada de $502 \mathrm{~m}$. Daí o Rio Mogi Guaçu percorre aproximadamente 31

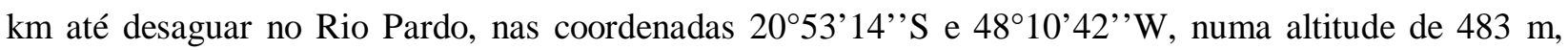
apresentando, desde sua foz até o início de seu trajeto, no município de Pitangueiras - SP, um desnível de 19 metros.

O mosaico não controlado obtido da área de estudo é exibido na Figura 2. Nesta, observa-se o trecho de $31 \mathrm{~km}$ do Rio Mogi Guaçu, na cor azul.

Figura 2 - Mosaico não controlado da área de estudo.

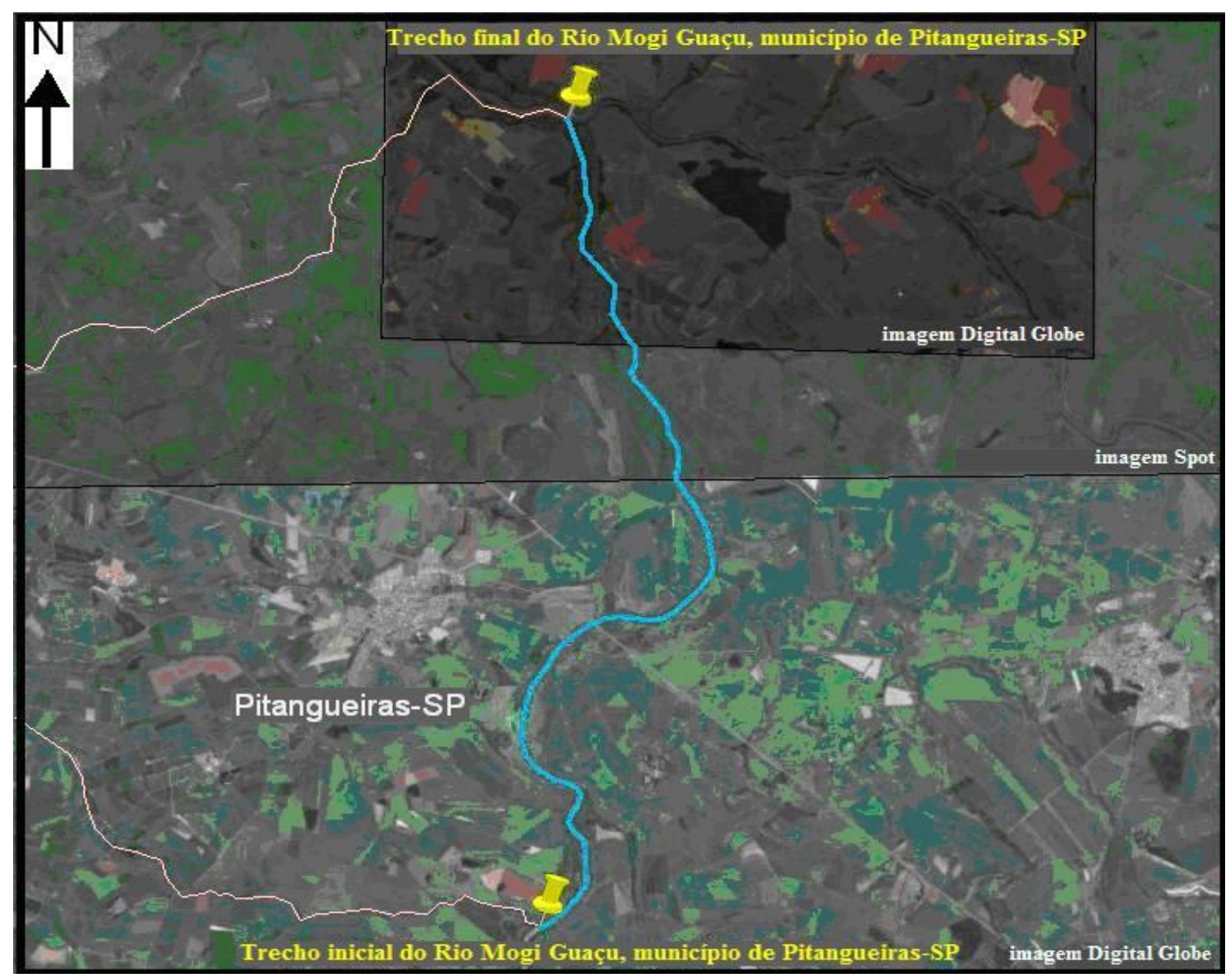

Nucleus, v.9, n.1, abr.2012 
Ampliando o mosaico da Figura 2, a Figura 3 exibe o início da área de estudo, que mostra as áreas de preservação permanente (cor verde) e as áreas invadidas pela agricultura nas áreas de preservação permanente (cor vermelha), no início do Rio Mogi Guaçu, município de Pitangueiras - SP.

Figura 3 - Delimitação das áreas de preservação permanente (APPs) e das áreas invadidas pela agricultura nas áreas de preservação permanente, trecho inicial do Rio Mogi Guaçu, município de Pitangueiras - SP.

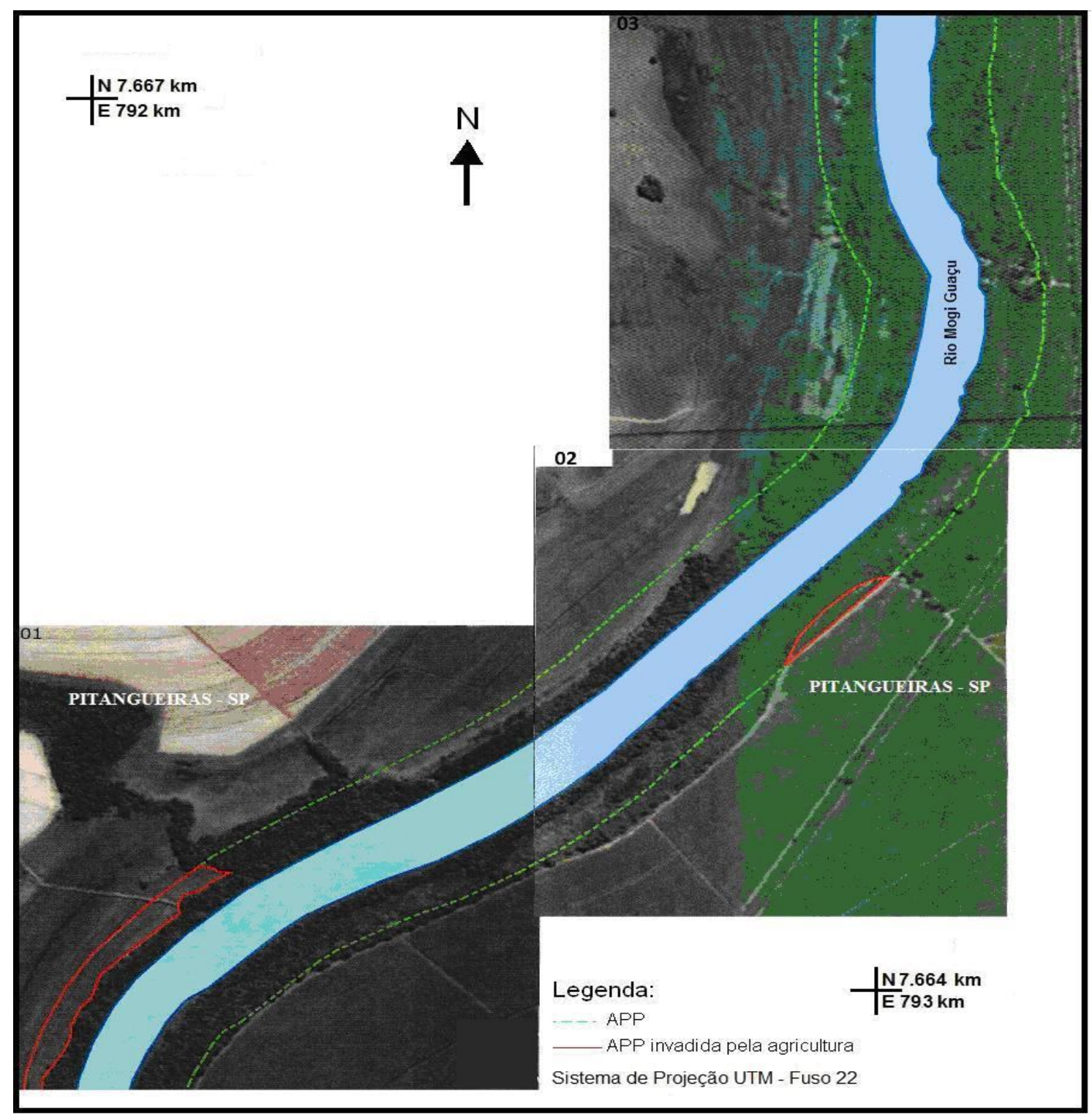

Na Figura 4, nota-se o deságue do Rio Mogi Guaçu no Rio Pardo. Ressalta-se que, neste trecho, as APPs consideradas foram somente as da margem esquerda do rio, pois a margem direita situa-se no município de Pontal - SP. 
Figura 4 - Delimitação das áreas de preservação permanente (APP) no trecho final do Rio Mogi Guaçu, município de Pitangueiras - SP.

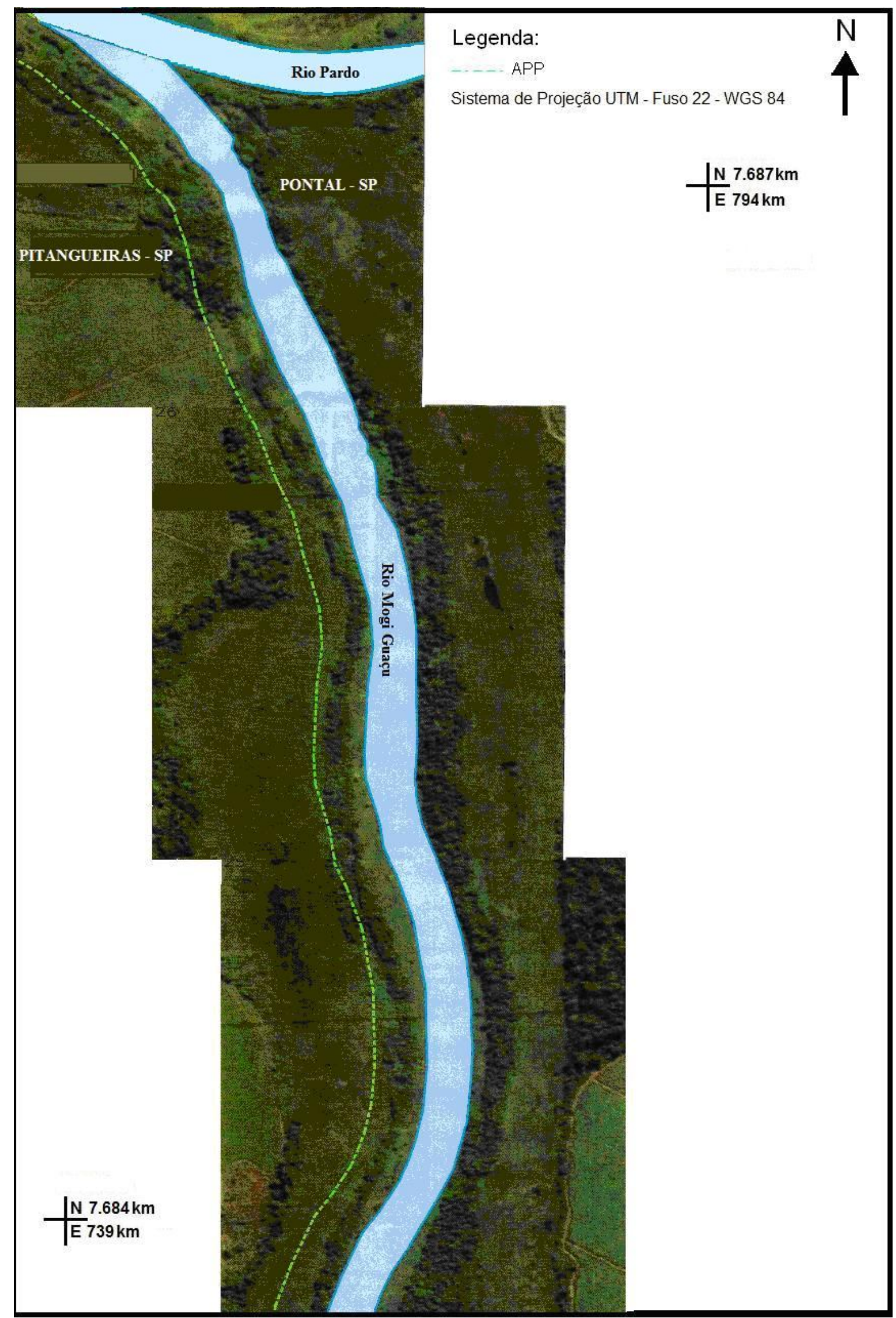


Nas 27 seções avaliadas, nota-se que a menor largura encontrada do Rio Mogi Guaçu é de $52 \mathrm{~m}$, e a maior largura é de $182 \mathrm{~m}$. Dessa forma, enquadra-se na categoria de 50 a $200 \mathrm{~m}$ de largura, tendo assim a obrigatoriedade de faixas de APPs de 100 metros para cada uma de suas margens a partir de seu curso d'água, conforme artigo $2^{\circ}$ da Lei 4.771/65 (MAGRI; BORGES, 2002).

Através de fotointerpretação visual, pode-se observar o tipo de vegetação das áreas de preservação permanente, como: vegetação arbórea, vegetação rasteira e área invadida pela agricultura. A Tabela 3 exibe os resultados desta classificação.

Tabela 3: Tipo de vegetação presente nas APPs ao longo do Rio Mogi Guaçu, município de Pitangueiras SP.

\begin{tabular}{|c|c|c|}
\hline Tipo de Vegetação & Área (ha) & $\%$ \\
\hline Arbórea & 275,8 & 64,2 \\
\hline Rasteira & 139,1 & 32,4 \\
\hline Área invadida pela agricultura & 14,4 & 3,4 \\
\hline Área Total & 429,3 & 100,0 \\
\hline
\end{tabular}

Dentre os 429,30 ha delimitados como área de preservação permanente ao longo do Rio Mogi Guaçu, município de Pitangueiras - SP, pode-se observar que existe uma extensa área de vegetação arbórea remanescente, com aproximadamente 275,7 ha, representando 64,2\% da área total estudada, seguido de 139,1 ha de área de vegetação rasteira, representando 32,4\% da área total estudada e 14,4 ha de área invadida pela agricultura, representando $3,4 \%$ da área total de estudo. Resultados similares foram encontrados em estudo realizado na bacia do Rio Uberaba - MG (VALLE JUNIOR et al., 2010).

\section{CONCLUSÃO}

Dentro das condições de avaliação, a área de preservação permanente invadida pela agricultura ao longo do Rio Mogi Guaçu, município de Pitangueiras-SP, é reduzida, visto que somente 3,4\% das APPs encontram-se utilizadas por atividade agrícola.

\section{REFERÊNCIAS}

BRASIL. Lei no 4.771, de 15 de setembro de 1965. Institui o novo Código Florestal. Brasília: DOU, 1965.

BRASIL. Resolução CONAMA n ${ }^{0}$ 303, de 20 de março de 2002. Dispõe sobre parâmetros, definições e limites de Áreas de Preservação Permanente. Brasília: DOU, 2002.

CAMPOS, S. P. Planejamento agroambiental de uma microbacia hidrográfica utilizando um Sistema de Informações Geográficas. 2001. 137 f. Tese (Doutorado em Agronomia/ Energia na Agricultura), Faculdade de Ciências Agronômicas, Universidade Estadual Paulista. Jaboticabal.

CBH-MOGI - COMITÊ DA BACIA HIDROGRÁFICA DO RIO MOGI GUAÇU. Proposta Plano da Bacia Hidrográfica do Rio Mogi Guaçu, período 2008-2011. Araras: CBH-MOGI, 2008. 
DIGITAL GLOBE. Imagem de satélite. Disponível em:

<http://browse.digitalglobe.com/imagefinder/showBrowseMetadata?catalogId=1010010001E9C904〉. Acesso em: 21 jul. $2011 \mathrm{~b}$.

DIGITAL GLOBE. Imagem de satélite. Disponível em:

<http://browse.digitalglobe.com/imagefinder/showBrowseMetadata?catalogId=1010010005C2B501>. Acesso em 21 jul. 2011 a.

GOOGLE EARTH. Disponível em: <htpp://earth.google.com/intl/pt-BR/>. Acesso em: 21 jul. 2011.

MAGRI, R. V. R.; BORGES, A. L. M. Vegetação de Preservação permanente e área urbana - uma interpretação do parágrafo único, do art. $2^{\circ}$, do Código Florestal. Revista de Direito Ambiental, São Paulo, 1996, ano I, v. 2.

OLIVEIRA, M. J. Proposta Metodológica para Delimitação Automática de Áreas de Preservação Permanente em Topos de Morro e em Linha de Cumeada. 2002. 53 f. Dissertação (Mestrado em Ciência Florestal) - Universidade Federal de Viçosa.

RIBEIRO, C. A. A. S. et al. Modelos digitais de elevação hidrologicamente consistentes para a Amazônia Legal. In: SIMPÓSIO DE RECURSOS HÍDRICOS DO CENTRO-OESTE, 2., 2002, Campo Grande. Anais... Campo Grande: 2002.

SOARES, V. P. et al. Avaliação das áreas de uso indevido da terra em uma microbacia no município de Viçosa - MG, através de fotografias aéreas e sistemas de informação geográfica. Revista Árvore, Viçosa, 2002, v.26, n.2, p.243-251.

SPOT. Imagem de satélite. Disponível em: http://www.spotimage.com/image/spot5/01jun2010 . Acesso em: 21 jul. 2010.

VALLE JUNIOR, R. F. et al. Determinação das áreas de preservação permanente na bacia Hidrográfica do Rio Uberaba - MG, utilizando o sistema de informação geográfica - SIG. Gl. Sci. Technol, , Rio Verde, 2010, v.3, n.1, p.19- 29.

ZANCOPÉ, M. H. de C.; PEREZ FILHO, A. Considerações a Respeito da Distribuição das Planícies Fluviais do Rio Mogi Guaçu. Revista Brasileira de Geomorfologia, Uberlândia, 2006, ano 7, n.1, p.6571. 